\title{
Towards Elucidation of the Role of Ubiquitination in the Pathogenesis of Parkinson's Disease with Semisynthetic Ubiquitinated $\alpha$-Synuclein**
}

\author{
Mirva Hejjaoui, Mahmood Haj-Yahya, K. S. Ajish Kumar, Ashraf Brik,* and Hilal A. Lashuel*
}

The ubiquitination of lysine residues has emerged as an important mechanism for the regulation of a variety of cellular processes, such as cell signaling, DNA repair, and protein degradation by the $26 \mathrm{~S}$ proteasome. ${ }^{[1,2]}$ Until recently, studies aimed at deciphering the effect of ubiquitination on protein function have relied primarily on biochemical approaches to reconstitute the ubiquitinated protein in vitro. As a result, progress in the field has been very much dependent on the discovery of the E2-E3 enzymatic machinery that is highly specific to the protein of interest. ${ }^{[3]}$ The lack of an effective and general method for the site-specific incorporation of ubiquitin (Ub) or polyubiquitin chains has hampered efforts to elucidate the molecular and structural basis underlying the effects of ubiquitination on the regulation of protein function. Several research groups have recently reported elegant chemical methods that facilitate site-specific peptide and protein ubiquitination. Several of these methods are based on the attachment of ubiquitin through non-native isopeptide bonds to generate enzymatically stable $\mathrm{Ub}$ conjugates of peptides or recombinant proteins. ${ }^{[4-9]}$ The site-specific ubiquitination of peptides and proteins through the formation of a native isopeptide bond is now possible as a result of studies by Muir and co-workers, Liu and co-workers, and our research group. ${ }^{[10-13]}$

We recently developed a highly efficient and chemoselective method to facilitate the site-specific ubiquitination of peptides and proteins. ${ }^{[12]}$ In our approach, a $\delta$-mercapto-

\footnotetext{
[*] M. Hejjaoui, ${ }^{[+]}$Prof. H. A. Lashuel

Brain Mind Institute

The Swiss Federal Institute of Technology EPFL

Station 19, 1015 Lausanne (Switzerland)

Fax: (+41) 21-693-9665

E-mail: hilal.lashuel@epfl.ch

Homepage: http://nmnf.epfl.ch

M. Haj-Yahya, ${ }^{[+]}$Dr. K. S. A. Kumar, Prof. A. Brik

Department of Chemistry

Ben-Gurion University of the Negev

Beer Sheva 84105 (Israel)

Fax: (+ 972) 8-647-2943

E-mail: abrik@bgu.ac.il

Homepage: http://www.bgu.ac.il/ abrik

['] These authors contributed equally.

$[\cdots *$ This research was supported by an ERC starting grant (H.L., M.H., 243182), the Edmond J. Safra Foundation, and partially supported by a Human Science Frontiers Young Investigator Grant (H.L. and A.B). We thank Dr. Sara Butterfield and Bruno Fauvet for insightful discussions.

(9) Supporting information for this article is available on the WWW under http://dx.doi.org/10.1002/anie.201005546.
}

lysine residue is used to mediate transthioesterification with an ubiquitin thioester, followed by S-N acyl transfer to form an isopeptide bond between ubiquitin and the lysine derivative. The thiol handle of $\delta$-mercaptolysine is then removed by desulfurization to furnish the desired native isopeptide linkage between ubiquitin and lysine without altering the native sequence of the target peptides/proteins. To facilitate the use of this unique residue in solid-phase peptide synthesis (SPPS) and sequential ligation, we also synthesized $\delta$ mercaptolysine with different protecting groups. ${ }^{[14]}$ Herein, we describe an efficient strategy based on the use of these (advanced) synthetic tools for the semisynthesis and characterization of $\alpha$-synuclein monoubiquitinated at Lys6 (T7-Ub$\alpha$-syn(K6)).

$\alpha$-Synuclein ( $\alpha$-syn) is a natively unfolded 140 amino acid presynaptic protein that is implicated in the pathogenesis of Parkinson's disease (PD) and related neurodegenerative diseases that are collectively termed "synucleinopathies". ${ }^{[15-17]}$ The pathology of PD is characterized by the loss of dopaminergic neurons and the presence of intracellular inclusions, known as Lewy bodies (LBs), which are composed primarily of $\alpha$-syn. ${ }^{[15]}$ Several posttranslational modifications, including phosphorylation, C-terminal truncations, and ubiquitination, have been shown to be closely associated with PD pathology and were identified in $\alpha$-syn within LBs isolated from PD patients brains. ${ }^{[18-21]}$ Understanding of the role of these modifications in the regulation of $\alpha$-syn aggregation, LB formation, and toxicity is crucial to the understanding of the biology of $\alpha$-syn and the elucidation of its role in the pathogenesis of PD, and may lead to the identification of novel therapeutic targets for the treatment of the disease.

The majority of $\alpha$-syn species found in Lewy bodies are mono- or diubiquitinated at multiple lysine residues. ${ }^{[18,19,21]}$ The directed site-specific ubiquitination of $\alpha$-syn at a single or multiple lysine residues has not been possible. For example, the coexpression of $\alpha$-syn with ubiquitin ligases results predominantly in mono- and diubiquitination at multiple sites. ${ }^{[22,23]}$ Similarly, the ubiquitination of recombinant monomeric or fibrillar $\alpha$-syn using rabbit reticulocytes fraction II or rat-brain extracts revealed that ubiquitination occurs at multiple but distinct lysine residues. ${ }^{[24]}$ Thus, it was not possible to investigate the effect of ubiquitination at specific lysine residues. To address this problem, we developed a semisynthetic strategy by combining cysteine- and $\delta$-mercaptolysine-based methods for native chemical ligation (NCL) ${ }^{[25]}$ for the site-specific incorporation of ubiquitin and the preparation of highly homogenous monoubiquitinated forms of $\alpha$-syn. 
We focused our efforts on $\alpha$-syn monoubiquitinated at K6 (T7-Ub- $\alpha$-syn(K6)) because in vitro ubiquitination of fibrillar $\alpha$-syn was shown to occur primarily at K6, K10, and K12. ${ }^{[24]}$ However, our approach can be applied to the preparation of monoubiquitinated $\alpha$-syn involving any lysine residues within the N-terminal region 1-18 and can be modified to enable ubiquitination at other lysine residues. The strategy for the semisynthesis of T7-Ub- $\alpha$-syn(K6) is based on expressed protein ligation (Scheme 1). Our design is based on the linking of two fragments by native chemical ligation (NCL): a recombinantly expressed $\alpha$-syn fragment comprising residues 19-140 and bearing an N-terminal Cys residue, $\alpha$-syn(19140), and a synthetic peptide thioester comprising the $\mathrm{N}$-terminal residues $1-18$ and bearing $\delta$-mercaptolysine, $\alpha$ syn(1-18)-SR, in which K6 is replaced with $\delta$-mercaptolysine protected with an acetamidomethyl (Acm) group. ${ }^{[14]}$ Notably, the sequence of $\alpha$-syn lacks Cys residues. Therefore, the ligation site was selected at Ala19, which is replaced temporarily with Cys to enable NCL and the assembly of full-length $\alpha$-syn. Cys19 is converted into Ala19 with simultaneous removal of the thiol handle of the $\delta$-mercaptolysine residue under desulfurization conditions to afford the native monoubiquitinated protein T7-Ub- $\alpha-\operatorname{syn}(\mathrm{K} 6) .{ }^{[26]}$ With this design in mind, the C-terminal fragment with an $\mathrm{N}$-terminal free cysteine residue, $\alpha$-syn(19-140), was expressed in Escherichia coli (see the Supporting Information). The $\alpha$-syn(1-18)SR peptide was prepared by SPPS according to the in situ neutralization protocol for tert-butoxycarbonyl (Boc) SPPS,${ }^{[27]}$ whereby the Acm-protected $\delta$-mercaptolysine residue was coupled instead of K6 (see the Supporting Information).

With both fragments in hand, we turned our attention to the ligation and ubiquitination steps. The NCL of $\alpha$-syn(19$140)$ and the synthetic $\alpha$-syn(1-18)-SR peptide thioester was carried out under denaturing conditions $(6 \mathrm{M}$ guanidine hydrochloride, $200 \mathrm{~mm}$ phosphate buffer, $\mathrm{pH} 7$ ) and in the presence of $2 \%(\mathrm{v} / \mathrm{v})$ thiophenol/phenylmethanethiol. The backbone ligation reaction was followed by reversed-phase

MDVVFMKGLSKAKEGVVAAAEKTKQGVAEAAGKTKEGVLYVGSKTKEGVVHGVATVAEKTKEQVTNVGGAVV TGVTAVAQKTVEGAGSIAAAATGFVKKDQLGKNEEGAPQEGILEDMPVDPDNEAYEMPSEEGYQDYEPEA ${ }^{14}$

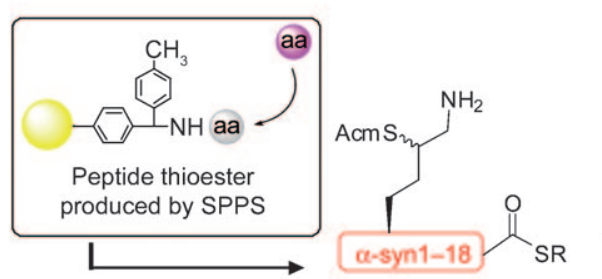

1
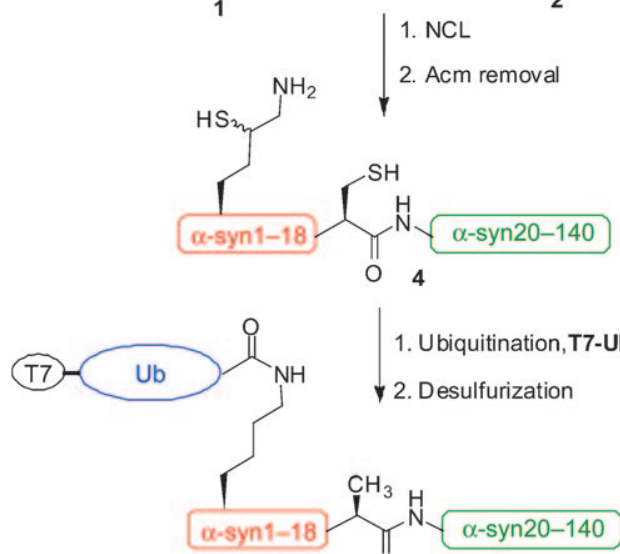

1. Ubiquitination, T7-Ub-SR

2. Desulfurization
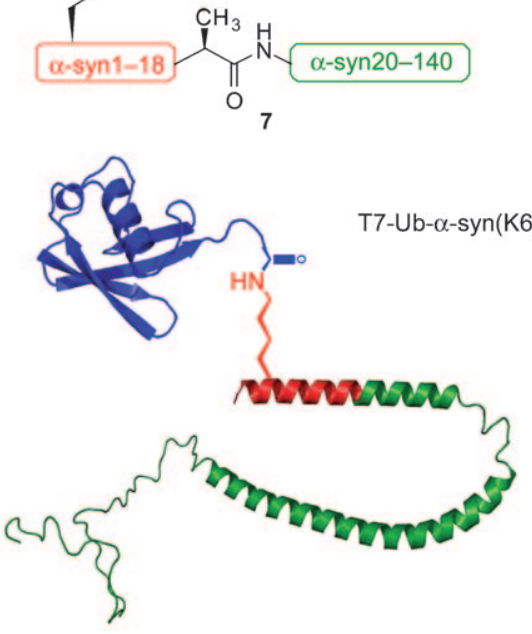

Scheme 1. Semisynthetic strategy for the ubiquitination of $\alpha$-syn at K6.
(RP) HPLC and mass spectrometry, both of which indicated near-complete ligation after 24 hours, and the desired product was isolated in $43 \%$ yield. Following the ligation step, the Acm protecting group was removed quantitatively by incubation with $\mathrm{Hg}(\mathrm{OAc})_{2}$ in $15 \%$ acetic acid for 3 hours, followed by treatment with dithiothreitol for an additional 12 hours (see the Supporting Information).

Next, we focused on the ubiquitination between the thioester T7-Ub-SR and fulllength $\alpha$-syn(1-140) presenting the free $\delta$-mercaptolysine residue. Under the ligation conditions described above, complete ligation of both proteins was observed after 12 hours, and the ubiquitinated product was isolated in $54 \%$ yield (see the Supporting Information). Finally, the product was subjected to the metal-free desulfurization conditions for 3 hours to give the native monoubiquitinated $\alpha$-syn T7-Ub- $\alpha-\operatorname{syn}(\mathrm{K} 6)$ in $78 \%$ yield (Figure 1 ). ${ }^{[28]}$

SDS-PAGE analysis of T7-Ub- $\alpha$-syn(K6) revealed a slow-migrating band at approximately $26 \mathrm{kDa}$; this band is consistent with the 


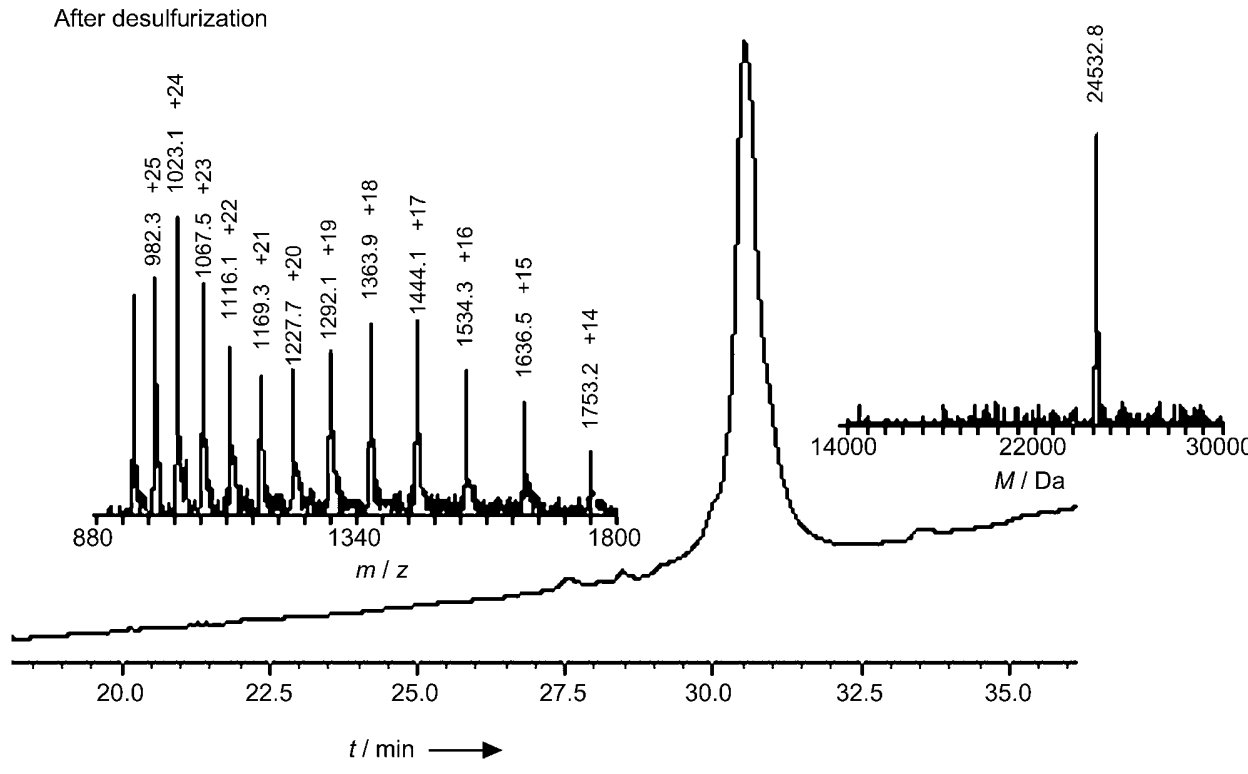

Figure 1. Analytical HPLC of the crude product of the desulfurization reaction with an observed mass of 24532.8 Da (calcd m/z: $24531.7 \mathrm{Da}$ ); this peak corresponds to the desired monoubiquitinated $\alpha$-syn. The reaction progress was analyzed by RP HPLC (C4 column) with a gradient of 5-60\% B (acetonitrile/1\% trifluoroacetic acid) over $30 \mathrm{~min}$. The reported mass is for total protein.

mass spectrometry data and the conjugation of one molecule of ubiquitin (Figure $2 \mathrm{a}$ ). This band was detectable by Western-blot (WB) analysis with anti-T7, anti- $\alpha$-syn, and antiubiquitin antibodies (Figure $2 \mathrm{a}$ ). To verify the formation of a native isopeptide bond between ubiquitin and $\alpha$-syn, we incubated T7-Ub- $\alpha$-syn(K6) with ubiquitin C-terminal-carboxy hydrolase (UCH-L3) to see if it could be hydrolyzed. Upon the addition of UCH-L3, we observed the generation of T7-ubiquitin monomers and wild-type (WT) $\alpha$-syn (see the Supporting Information).

Having verified the chemical integrity of T7-Ub- $\alpha-$ $\operatorname{syn}(\mathrm{K} 6)$, we focused on elucidating the effect of ubiquitination on the secondary structure and the membrane-binding and aggregation properties of $\alpha$-syn in vitro by using circular dichroism (CD), a thioflavin $\mathrm{T}$ (ThT) binding assay, and transmission electron microscopy (TEM). As expected, WT $\alpha$-syn exhibited a CD spectrum consistent with a random-coil structure, whereas T7-Ub-SR showed a spectrum that reflected a mixture of $\alpha$ helices, $\beta$ sheets, and random coils and was thus consistent with the structure of ubiquitin. ${ }^{[29]}$ The $\mathrm{CD}$ spectrum of T7-Ub- $\alpha$-syn(K6) was virtually identical to that obtained for a solution containing an equimolar mixture of T7-Ub-SR and WT $\alpha$-syn. This result suggests that the native structures of ubiquitin and $\alpha$-syn are preserved in the ubiquitinated $\alpha$-syn, T7-Ub- $\alpha$-syn(K6).

In neurons, $\alpha$-syn is localized in the cytoplasm and the membrane. The $\mathrm{N}$-terminal region comprising residues 1 to about $100^{[30-32]}$ has been shown to mediate the interaction of $\alpha$-syn with membranes and to adopt an $\alpha$-helical structure upon interaction with lipid vesicles in vitro.

The effect of ubiquitination on $\alpha$-syn membrane binding has not been investigated. WT $\alpha$-syn and monoubiquitinated $\alpha$-syn adopt a similar $\alpha$-helical structure upon binding to 1-palmitoyl-2-oleoyl-sn-glycero-3-phospho-(1'-rac-glycerol)
(POPG) vesicles (Figure 2b), which suggests that the conjugation of a ubiquitin moiety on K6 does not induce major changes in the structure of $\alpha$-syn in solution or alter its interaction with synthetic lipids.

To determine the effect of $\mathrm{N}$-terminal ubiquitination on $\alpha$-syn fibril formation, we compared the fibrilization of the $\alpha$-syn monoubiquitinated at K6 to that of the WT protein by using the ThT fluorescence assay and TEM. Ubiquitination at $\mathrm{K} 6$ resulted in significant inhibition of $\alpha$-syn fibril formation, as evidenced by the absence of any change in the ThT signal after incubation with rotation at $37^{\circ} \mathrm{C}$ for 10 days (Figure $2 \mathrm{c}$ ). In agreement with the ThT data, WT $\alpha$-syn formed extensive mature fibrillar structures, whereas no fibrils were detected in samples containing the monoubiquitinated protein (Figure $2 \mathrm{~d}$ ). These findings are consistent with previous reports that not all $\alpha$-syn inclusions in transgenic mouse models are ubiquitinated. ${ }^{[21-33]}$ Thus, ubiquitination does not appear to be required for inclusion formation and could be a late event that occurs after synuclein fibrilization. ${ }^{[21]}$ On the other hand, our data contradict the results of other studies indicating that ubiquitination enhances $\alpha$-syn aggregation in vitro and in cell cultures. ${ }^{[22,23]}$ However, none of these studies examined the aggregation of homogenous preparations of monoubiquitinated $\alpha$-syn; instead, the samples used contained heterogeneous mixtures of unmodified $\alpha$-syn and $\alpha$-syn ubiquitinated at different lysine residues.

The semisynthesis of $\alpha$-syn provides unique opportunities to explore cross-talk between different posttranslational modifications and how it influences $\alpha$-syn aggregation and LB formation in synucleinopathies. For example, several studies have found a close association between ubiquitination and the phosphorylation of $\alpha$-syn at S129 within LBs $;^{[18-20]}$ this association suggests that S129 phosphorylation may play a role in the regulation of $\alpha$-syn ubiquitination and degradation. Studies by Nonaka et al. suggested that the effect of S129 phosphorylation on $\alpha$-syn ubiquitination in vitro is minor. ${ }^{[24]}$ However, the effect of ubiquitination at each of the putative lysine residues on $\alpha$-syn phosphorylation has not been investigated.

To explore the interplay between these two modifications, we investigated the effect of ubiquitination at K6 on the extent of $\alpha$-syn phosphorylation at S87 and S129 by three kinases, caseine kinase I, polo-like kinase 3, and G-proteincoupled receptor kinase 5 , which are known to phosphorylate $\alpha$-syn at these residues. ${ }^{[34-37]}$ We took advantage of the difference in size between WT and T7-Ub- $\alpha-\operatorname{syn}(\mathrm{K} 6)$ and 
a)

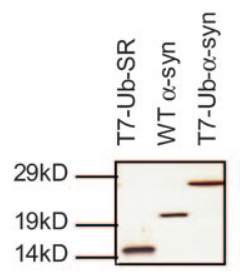

b)

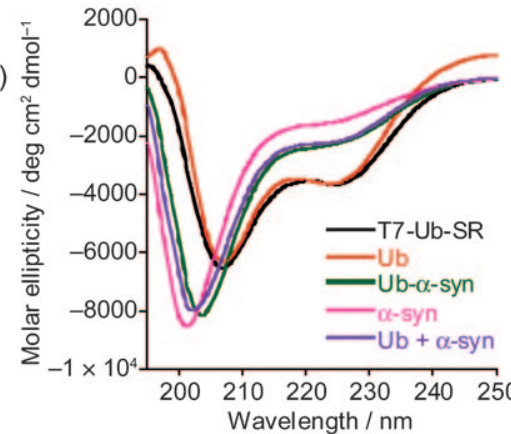

c)

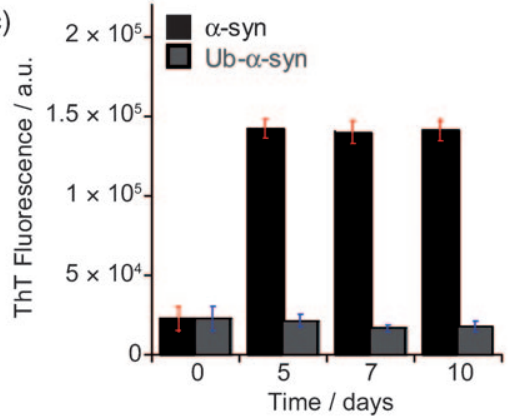

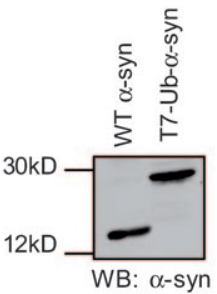
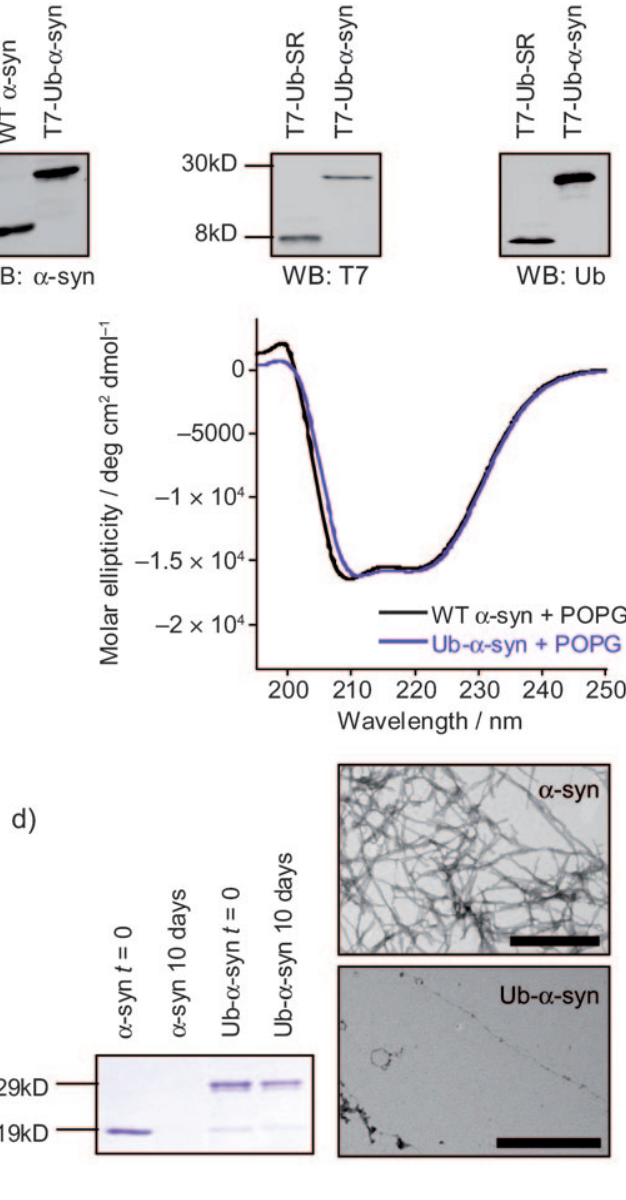

Figure 2. Characterization of $\alpha$-syn monoubiquitinated at K6. a) SDS-PAGE analysis ( $15 \%$ gel, silver staining) of T7-Ub-SR, WT $\alpha$-syn, and T7-Ub- $\alpha$-syn. Immunoblots of T7-Ub-SR and T7-Ub- $\alpha$-syn with anti-T7 and antiubiquitin antibodies (Zymed) and of WT $\alpha$-syn and T7-Ub- $\alpha$-syn with an anti- $\alpha$-syn antibody. b) CD spectra of T7-Ub-SR, ubiquitin (Sigma), a mixture of ubiquitin and WT $\alpha$-syn, WT $\alpha$-syn, and T7-Ub- $\alpha$-syn. CD spectra of WT $\alpha$-syn and T7-Ub- $\alpha$-syn bound to POPG vesicles. c) ThT fluorescence assay of samples after $0(t=0), 5,7$, and 10 days. d) Quantification of monomeric $\alpha$-syn by SDS-PAGE analysis at $t=0$ and 10 days after filtration through a $100 \mathrm{kDa}$ membrane. TEM images of WT $\alpha$-syn and T7-Ub- $\alpha$-syn at day 7; scale bar: $200 \mathrm{~nm}$. The figures are representative of two independent experiments.

included both proteins in the phosphorylation reaction to assess the activity of the kinases and enable direct comparison of the two proteins under identical phosphorylation conditions. We found that ubiquitination at K6 does not influence significantly the extent of $\alpha$-syn phosphorylation at S87 (by CK1) and S129 (by CK1, GRK5, and PLK3; see the Supporting Information).

In summary, we have described the semisynthesis and characterization of a site-specifically (K6) monoubiquitinated form of $\alpha$-syn (T7-Ub- $\alpha$-syn(K6)). The strategy yielded sufficient quantities that enabled us to perform biochemical and biophysical studies to elucidate the effect of this modification on the structure, membrane binding, and fibrilization of $\alpha$-syn. Furthermore, we were able to investigate the cross-talk between two disease-associated posttranslational modifications (ubiquitination and phosphorylation at specific residues). The ability to introduce ubiquitin modifications site-specifically into $\alpha$-syn represents a major

advance towards the elucidation of the role of ubiquitination in regulating the function(s) of $\alpha$-syn in health and disease. Our findings present strong evidence in support of the hypothesis that the N-terminal ubiquitination of $\alpha$-syn stabilizes the monomeric form of the protein and thus prevents its oligomerization and fibrillogenesis in vitro.

The results presented herein highlight the potential of applying advances in the semisynthesis of proteins to dissect the role of posttranslational modifications in modulating $\alpha$-syn function in health and disease. Current efforts in our laboratories are focused on elucidating the effect of ubiquitination at other lysine residues as well as the effect of other posttranslational modifications. ${ }^{[38]}$ We are also investigating the consequences of these modifications on the subcellular localization, lifetime, and toxicity of $\alpha$-syn in cellular models of synucleinopathies.

Received: September 4, 2010

Published online: December 10, 2010

Keywords: aggregation .

Parkinson's disease · proteins .

semisynthesis · ubiquitination

[1] C. M. Pickart, D. Fushman, Curr. Opin. Chem. Biol. 2004, 8, 610-616.

[2] F. Ikeda, I. Dikic, EMBO Rep. 2008, 9, 536-542.

[3] C. M. Pickart, Annu. Rev. Biochem. 2001, 70, 503-533.

[4] C. Chatterjee, R. K. McGinty, B. Fierz, T. W. Muir, Nat. Chem. Biol. 2010, 6, 267-269.

[5] J. Chen, Y. Ai, J. Wang, L. Haracska, Z. Zhuang, Nat. Chem. Biol. 2010, 6, 270-272.

[6] A. Shanmugham, A. Fish, M. P. A. Luna-Vargas, A. C. Faesen, F. El Oualid, T. K. Sixma, H. Ovaa, J. Am. Chem. Soc. 2010, 132, $8834-8835$.

[7] N. D. Weikart, H. D. Mootz, ChemBioChem 2010, 11, 774-777.

[8] X. Li, T. Fekner, J. J. Ottesen, M. K. Chan, Angew. Chem. 2009, 121, 9348-9351; Angew. Chem. Int. Ed. 2009, 48, 9184-9187.

[9] L. Yin, B. Krantz, N. S. Russell, S. Deshpande, K. D. Wilkinson, Biochemistry 2000, 39, $10001-10010$.

[10] C. Chatterjee, R. K. McGinty, J. P. Pellois, T. W. Muir, Angew. Chem. 2007, 119, 2872-2876; Angew. Chem. Int. Ed. 2007, 46, 2814-2818.

[11] R. K. McGinty, J. Kim, C. Chatterjee, R. G. Roeder, T. W. Muir, Nature 2008, 453, 812-816.

[12] K. S. Ajish Kumar, M. Haj-Yahya, D. Olschewski, H. A. Lashuel, A. Brik, Angew. Chem. 2009, 121, 8234-8238; Angew. Chem. Int. Ed. 2009, 48, 8090-8094. 
[13] R. Yang, K. K. Pasunooti, F. Li, X. W. Liu, C. F. Liu, J. Am. Chem. Soc. 2009, 131, 13592-13593.

[14] M. Haj-Yahya, K. S. Ajish Kumar, L. A. Erlich, A. Brik, Biopolymers 2010, 94, 504-510.

[15] M. G. Spillantini, T. D. Bird, B. Ghetti, Brain Pathol. 1998, 8, $387-402$.

[16] B. C. V. Campbell, C. A. McLean, J. G. Culvenor, W. P. Gai, P. C. Blumbergs, P. Jäkälä, K. Beyreuther, C. L. Masters, Q.-X. Li, J. Neurochem. 2001, 76, 87-96.

[17] W. P. Gai, J. H. Power, P. C. Blumbergs, J. G. Culvenor, P. H. Jensen, J. Neurochem. 1999, 73, 2093-2100.

[18] J. P. Anderson, D. E. Walker, J. M. Goldstein, R. de Laat, K. Banducci, R. J. Caccavello, R. Barbour, J. Huang, K. Kling, M. Lee, L. Diep, P. S. Keim, X. Shen, T. Chataway, M. G. Schlossmacher, P. Seubert, D. Schenk, S. Sinha, W. P. Gai, T. J. Chilcote, J. Biol. Chem. 2006, 281, 29739-29752.

[19] M. Hasegawa, H. Fujiwara, T. Nonaka, K. Wakabayashi, H. Takahashi, V. M. Lee, J. Q. Trojanowski, D. Mann, T. Iwatsubo, $J$. Biol. Chem. 2002, 277, 49071-49076.

[20] G. K. Tofaris, A. Razzaq, B. Ghetti, K. S. Lilley, M. G. Spillantini, J. Biol. Chem. 2003, 278, 44405-44411.

[21] D. M. Sampathu, B. I. Giasson, A. C. Pawlyk, J. Q. Trojanowski, V. M. Lee, Am. J. Pathol. 2003, 163, 91-100.

[22] R. Rott, R. Szargel, J. Haskin, V. Shani, A. Shainskaya, I. Manov, E. Liani, E. Avraham, S. Engelender, J. Biol. Chem. 2008, 283, $3316-3328$.

[23] J. T. Lee, T. C. Wheeler, L. Li, L. S. Chin, Hum. Mol. Genet. 2008, 17, 906 - 917 .

[24] T. Nonaka, T. Iwatsubo, M. Hasegawa, Biochemistry 2005, 44, $361-368$.

[25] P. E. Dawson, T. W. Muir, I. Clark-Lewis, S. B. Kent, Science 1994, 266, 776-779.

[26] L. Z. Yan, P. E. Dawson, J. Am. Chem. Soc. 2001, 123, 526-533.

[27] M. Schnolzer, P. Alewood, A. Jones, D. Alewood, S. B. Kent, Int. J. Pept Protein Res. 1992, 40, 180-193.

[28] Q. Wan, S. J. Danishefsky, Angew. Chem. 2007, 119, 9408-9412; Angew. Chem. Int. Ed. 2007, 46, 9248-9252.
[29] J. Jenson, G. Goldstein, E. Breslow, Biochim. Biophys. Acta Protein Struct. 1980, 624, 378-385.

[30] C. R. Bodner, A. S. Maltsev, C. M. Dobson, A. Bax, Biochemistry 2010, 49, 862-871.

[31] T. S. Ulmer, A. Bax, N. B. Cole, R. L. Nussbaum, J. Biol. Chem. 2005, 280, $9595-9603$.

[32] M. Bisaglia, I. Tessari, L. Pinato, M. Bellanda, S. Giraudo, M. Fasano, E. Bergantino, L. Bubacco, S. Mammi, Biochemistry 2005, 44, 329-339.

[33] H. van der Putten, K. H. Wiederhold, A. Probst, S. Barbieri, C. Mistl, S. Danner, S. Kauffmann, K. Hofele, W. P. Spooren, M. A. Ruegg, S. Lin, P. Caroni, B. Sommer, M. Tolnay, G. Bilbe, $J$. Neurosci. 2000, 20, 6021-6029.

[34] M. K. Mbefo, K. E. Paleologou, A. Boucharaba, A. Oueslati, H. Schell, M. Fournier, D. Olschewski, G. Yin, M. Zweckstetter, E. Masliah, P. J. Kahle, H. Hirling, H. A. Lashuel, J. Biol. Chem. 2010, 285, 2807-2822.

[35] S. Arawaka, M. Wada, S. Goto, H. Karube, M. Sakamoto, C. H. Ren, S. Koyama, H. Nagasawa, H. Kimura, T. Kawanami, K. Kurita, K. Tajima, M. Daimon, M. Baba, T. Kido, S. Saino, K. Goto, H. Asao, C. Kitanaka, E. Takashita, S. Hongo, T. Nakamura, T. Kayama, Y. Suzuki, K. Kobayashi, T. Katagiri, K. Kurokawa, M. Kurimura, I. Toyoshima, K. Niizato, K. Tsuchiya, T. Iwatsubo, M. Muramatsu, H. Matsumine, T. Kato, J. Neurosci. 2006, 26, 9227-9238.

[36] M. Okochi, J. Walter, A. Koyama, S. Nakajo, M. Baba, T. Iwatsubo, L. Meijer, P. J. Kahle, C. Haass, J. Biol. Chem. 2000, 275, 390-397.

[37] K. E. Paleologou, A. Oueslati, G. Shakked, C. C. Rospigliosi, H.Y. Kim, G. R. Lamberto, C. O. Fernandez, A. Schmid, F. Chegini, W. P. Gai, D. Chiappe, M. Moniatte, B. L. Schneider, P. Aebischer, D. Eliezer, M. Zweckstetter, E. Masliah, H. A. Lashuel, J. Neurosci. 2010, 30, 3184-3198.

[38] A. Oueslati, M. Fournier, H. A. Lashuel, Prog. Brain Res. 2010, $183,115-145$. 